\title{
Chronic Pelvic Pain and Irritable Bowel Syndrome: Is Subclinical Inflammation Bridging the Gap?
}

\author{
DOINA GEORGESCUㄹ, MIRCEA STEFAN IURCIUC2*, IZABELLA PETRE ${ }^{3}$, LIVIU ANDREI GEORGESCU4, FLORIN SZASZ5 \\ IOANA IONITA ${ }^{1}$, OANA ELENA ANCUSA ${ }^{1}$, MIHAI IONITA ${ }^{1}$, DANIEL LIGHEZAN ${ }^{1}$ \\ ${ }^{1}$ Victor Babes University of Medicine and Pharmacy,, Department of Internal Medicine, 2 Eftimie Murgu Sq., 300041, Timisoara, \\ Romania \\ 2 Victor Babes University of Medicine and Pharmacy, Department of Cardiology, 2 Eftimie Murgu Sq., 300041, Timisoara, Romania \\ ${ }^{3}$ Victor Babes University of Medicine and Pharmacy, Department of Obstetrics and Gynecology, 2 Eftimie Murgu Sq., 300041, \\ Timisoara, Romania \\ ${ }^{4}$ Victor Babes University of Medicine and Pharmacy, Department of Urology, 2 Eftimie Murgu Sq., 300041, Timisoara, Romania \\ ${ }^{5}$ University of Oradea, 1 Universitatii Str., 410087, Oradea, Romania
}

\begin{abstract}
Irritable bowel syndrome (IBS) is characterized by a multitude of symptoms digestive and extra- digestive that need at some point a multidisciplinary approach. This study aimed at profiling IBS associated with chronic pelvic pain (CPP) in young females. A cross sectional observatory study on 40 consecutive young female patients (under 45 years) with IBS (Rome III) was performed. Patients were assigned in two groups, as matched pairs, based on the presence of chronic pelvic pain (CPP) symptoms: cystalgia, urinary urge and dyspareunia: $C P P(+)$ vs. $C P P(-)$ and undertook clinical examinations with special protocols related to migraine disability, fibromyalgia, temporo-mandibular joint dysfunction, as well as assessment of anxiety and severity of abdominal pain. Laboratory work-up (blood, urine and stool) as well as multiple exams: digestive endoscopy, abdominal and pelvic ultrasound//CT were performed. Results: CPP (+) group displayed higher CRP, TNF-alpha, gut dysbiosis (DB) and abdominal pain severity, as well as associated fibromyalgia, migraine and anxiety mood disorder. DB positively correlated with inflammatory markers and symptoms characterizing CPP. In conclusion, young female IBS patients with concurrent CPP symptoms often experienced other associated functional pain conditions like FM and migraine along with anxiety, more severe abdominal complaints as well as higher gut $D B$ and consecutively subclinical pro-inflammatory status. Strong positive correlations of gut DB to inflammatory markers as well as to CPP symptoms give the relationship IBS-CPP a new perspective.
\end{abstract}

Keywords: CPP, IBS, inflammation, gut dysbiosis

Irritable bowel syndrome (IBS), known as functional intestinal condition is characterized by a multitude of symptoms that need at some point a multidisciplinary approach, either diagnostic or therapeutic $[1,2]$.

Apart from typical symptoms such as abdominal pain, bloating, intestinal habit disturbances (constipation, diarrhea, alternant constipation/diarrhea, sensation of incomplete defecation), a large co-existing extra digestive pathology is often reported in IBS patients [3]. Patients with IBS, sometimes long before confirmation of gut condition, have a history of headaches, fibromyalgia(FM), temporomandibular joint disorder(TMJD), not to mention mood disorders like anxiety and depression. Since there are patients, especially women, complained of multiple pain of no organic or with unknown origin, some authors labeled this condition as functional pain syndrome, but a clear understanding of underlying pathways still lacks [4]. Gastrointestinal motility disturbances, visceral hyperalgesia, corrupted brain-gut dialogue, as well as gut dysbiosis (DB) are incriminated as possible causes of IBS [5]. Female patients with IBS often reported associated extra-digestive symptoms consistent with chronic pelvic pain (CPP) condition, that sometimes make the first visit to gynecologist and not to gastroenterologist, while symptoms somehow overlap gynecological pathology [6].

\section{Experimental part}

Selection of candidates for study: 40 female patients with confirmed IBS (Rome III), aged under 45 years were consecutively recruited during hospitalization and freely joined this cross-sectional, exploratory study. They were assigned into 2 equal groups, as matched pairs, based upon the presence or absence of CPP (EAU, 2014):20 patients CPP negative vs. 20 patients CPP positive. A lot of diseases and conditions were ruled out as a result of an integrated, interdisciplinaryapproach.

Exclusion criteria Patients with chronic organ insufficiency (respiratory, heart, liver or kidney), obesity( BMI over $30 \mathrm{~kg} / \mathrm{m}^{2}$ ), diabetes mellitus, thyroid or collagen diseases, visceral cancer, hematological or hematooncological conditions, associated inflammatory bowel disease (IBD), celiac disease or gluten sensitivity, treatment with antibiotics or probiotics, as well as those having gynecological or urological issues, were excluded from this study.

Patients undertook a thoroughly clinical examination, with the assessment of concurrent pains: $F M$, headaches (MIDAS-migraine disability assessment), TMJ D and generalized anxiety disorder (GAD). Urinary urge, cystalgia and dyspareunia were scored using a scale ranging from $0=$ absent to $6=$ very severe and a global evaluation of abdominal discomfort ( $1=$ mild, $2=$ moderate, $3=$ severe), was performed as well. Blood and urine biochemistry work-up, urine cytology and microbiology, as well as stool microbiology exams were performed using standard, accredited laboratory methods. Gut dysbiosis (DB) was semiquantitative assessed as: $0=$ absent, $1=$ mild, $2=$ medium, $3=$ severe. Digestive endoscopies, abdominal and pelvic ultrasound, as well as pelvic CT were performed.

*email: mirceaiurciuc@gmail.com; Phone: +40723684748 
IBS was diagnosed according to Rome III criteria: 3 months of continuous or recurrent symptoms of abdominal pain or discomfort eventually relieved with bowel movements, associated or not with changes in frequency of defecation or in stool consistency. Two or more of the following are present at least $25 \%$ of the time: change in stool frequency ( $>3$ bowel movements per day or $<3$ per week); obvious differences in stool appearance (hard, loose, watery or poorly formed stools); presence of mucus in stools; bloating and feeling of abdominal distension; or sensation of incomplete evacuation, straining, or urgency [7]. CPP diagnostic criteria in women patients relied on persistent pain in structures related to the pelvis, often associated with negative cognitive, behavioral, sexual and emotional consequences, as well as various symptoms suggesting lower urinary tract, bowel, pelvic floor or gynecological issues, according to EAU, 2014 [8]. Migraine diagnostic and severity assessment were based on international headache consensus IHC-2 and migraine disability MIDAS scores: 0-5 as minimal, 8-10 as mild, 1120 as moderate, and 21 or more, as severe disability $[9,10]$. GAD was scored minor, under 5, moderate, 6-10 and severe, 11-21[11]. TMJ D was assessed as present or absent [12]. FM assessment was based on a protocol examination of painful points, according to ACR, 2010 and scored as 1,minor, 2,moderate and 3 severe [13]. All subjects gave their informed consent for inclusion before they participated in the study. The study was conducted in accordance with the Declaration of Helsinki. Graph Pad Prism 8 software (Graph Pad Software, Inc., La J olla, CA, USA) was used to perform statistical analysis. Quantitative variables were expressed as mean values $(\mathrm{MV}) \pm$ standard deviation (SD) and qualitative variables were expressed as percentages. Chi-squared test for comparison of two groups, in cases of categorical analyzed variables, as well as unpaired t test were performed; $p$ values were interpreted with confidence interval $\mathrm{Cl}=95 \%, \mathrm{p} \leq 0.05$ being considered statistically significant. Nonparametric Spearman correlation test with the calculation of $r$ coefficient and consecutively linear regression equation were realized. Graph representation was performed accordingly.

\section{Results and discussions}

Demographic and biological baseline data are depicted in Table 1. There were no statistically significant differences related to age or location, marital status, education, CBC and standard biochemistry tests. However, inflammatory markers like HS-CRP $(p=0.0132)$ and TNF-alpha $(p=0.001)$, as well as DB ( $p=0.0001)$ were statistically significant higher in CPP positive group.

Clinical characteristics in study population are summarized in Table 2. There were no statistical differences concerning alcohol drinking, cigarettes smoking, oral contraception, occupational stress or previous pelvic surgery. Statistically significant differences were observed in CPP positive group related to more severe scores for abdominal pain $(p=0.0025), F M(p=0.0001)$, MIDAS $(p=0.05)$ and $\operatorname{GAD}(p=0.03)$.

Each symptom characterizing CPP associated to IBS was separately analyzed concerning possible link to top clinical and biological parameters which at initially descriptive data distribution demonstrated a significant statistical difference. As seen in Figure 1, cystalgia was strong positive correlated to abdominal pain $(r=0.59$; $p=0.005)$ and gut $D B(r=0.44 ; p=0.04)$

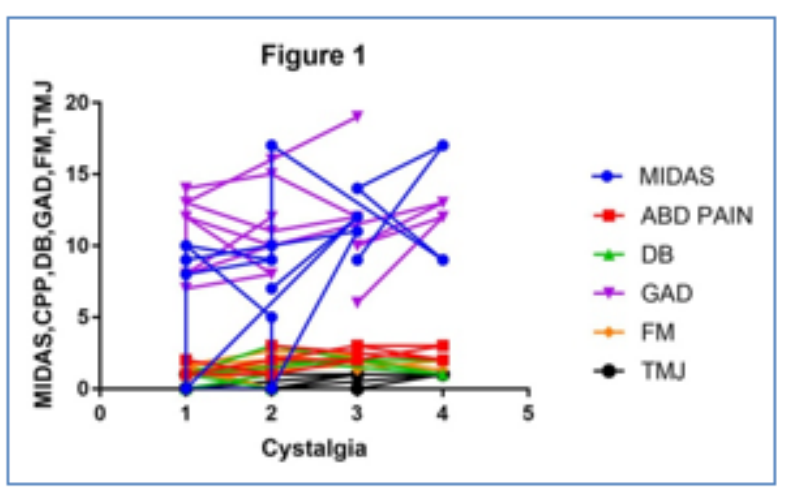

Fig. 1 Study of cystalgia correlations

\begin{tabular}{|c|c|c|c|c|}
\hline & Baseline data & $\mathrm{CPP}(+)$ & CPP(-) & $\mathbf{p}$ \\
\hline 1 & Age (years) & $37,45 \pm 4,94$ & $38,30 \pm 3,79$ & 0,5448 \\
\hline 2 & Location U/R & $80 \% / 20 \%$ & $60 \% / 40 \%$ & 0,1730 \\
\hline 3 & Marital situation(married) & $80 \%$ & $85 \%$ & 0,6812 \\
\hline 4 & High education & $60 \%$ & $70 \%$ & 0,5127 \\
\hline 5 & Hemoglobin(g/dl) & $11,930=0,353$ & $12,216=0,348$ & 0,0152 \\
\hline 6 & Leukocytes $/ \mathrm{mm}^{3}$ & $7,16 \times 10^{3}=0,235 \times 10^{3}$ & $7,125 \times 10^{3}=0,215 \times 10^{3}$ & 0,6258 \\
\hline 7 & $\mathrm{HS}-\mathrm{CRP}(\mu \mathrm{g} / \mathrm{mL})$ & $0,250 \pm 0,167$ & $0,145 \pm 0,069$ & 0,0132 \\
\hline 8 & TNF-alpha (pg/ml) & $7,465 \pm 2,018$ & $4,975 \pm 1,121$ & 0,001 \\
\hline 9 & Creatinine $(\mathrm{mg} / \mathrm{dL})$ & $1,042=1,203$ & $0,740 \pm 0,088$ & 0,2700 \\
\hline 10 & $\mathrm{ALT}(\mathrm{IU} / \mathrm{ml})$ & $22,85 \pm 3,59$ & $22,55 \pm 3,63$ & 0,7942 \\
\hline 11 & Blood sugar(mg/dL) & $86,25 \pm 5,18$ & $83,90 \pm 7,85$ & 0,2707 \\
\hline 12 & DB scoring & $1,4 \pm 0,75$ & $0,45 \pm 0,5$ & 0,0001 \\
\hline
\end{tabular}

Table 1

DEMOGRAPHIC AND BIOLOGIC BASELINE DATA ON RESEARCH PARTICIPANTS

Legend $U / R=$ urban/rural, $H S-C R P=$ high specific- $C$ reactive protein, $T N F=$ tumor necrosis factor, 


\begin{tabular}{|c|l|c|c|c|}
\hline & Clinical characteristics & CPP(+) & CPP(-) & P \\
\hline $\mathbf{1}$ & Alcohol & $25 \%$ & $20 \%$ & 0,7085 \\
$\mathbf{2}$ & Smoking & $55 \%$ & $50 \%$ & 0,7546 \\
$\mathbf{3}$ & Oral contraception & $15 \%$ & $25 \%$ & 0,4350 \\
$\mathbf{5}$ & Occupational stress & $90 \%$ & $80 \%$ & 0,3819 \\
$\mathbf{6}$ & Previous pelvic surgery & $15 \%$ & $5 \%$ & 0,2980 \\
$\mathbf{7}$ & Abdominal pain score & $1,80 \pm 0,77$ & $1,00 \pm 0,79$ & 0,0025 \\
$\mathbf{8}$ & Migraine (MIDAS) & $\mathbf{8 , 3 \pm 5 , 1 5}$ & $5,30 \pm 1,16$ & 0,05 \\
$\mathbf{9}$ & TMJD presence & $65 \%$ & $35 \%$ & 0,06 \\
\hline $\mathbf{1 0}$ & GAD score & $10,8 \pm 3,68$ & $8,7 \pm 2,25$ & 0,03 \\
\hline
\end{tabular}

Table 2

CLINICAL CHARACTERISTICS IN RESEARCH PARTICIPANTS

Legend: $\mathrm{FM}=$ fibromyalgia, $\mathrm{MIDAS}=$ migraine disability assessment, $\mathrm{TMJ} \mathrm{D}=$ temporomandibular joint disorder, $\mathrm{GAD}=$ generalized anxiety disorder.

Study of dyspareunia correlations are depicted in Figure 2. We noted a strong positive correlation of dyspareunia to FM $(r=0.48 ; p=0.03)$ and gut $D B(r=0.48 ; p=0.02)$.

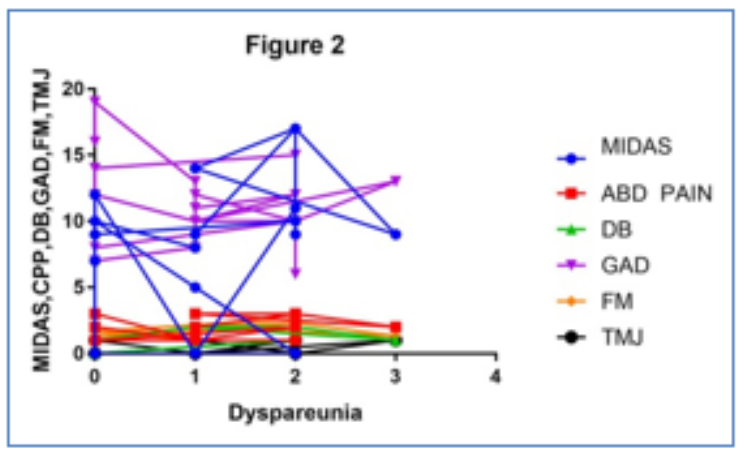

Fig. 2 Study of dyspareunia correlations

As illustrated in Figure 3 urinary urge was strong correlated to FM $(r=0.45 ; p=0.04)$ as well as to gut $D B$ $(r=0.53 ; p=0.01)$.

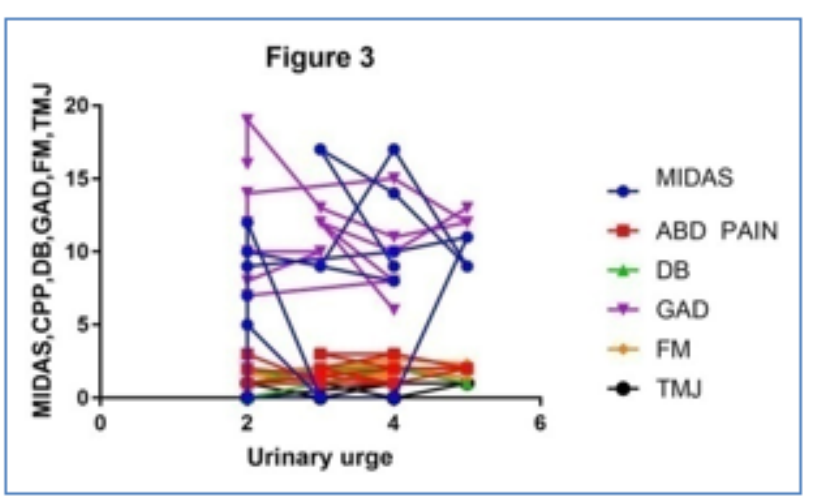

Fig.3 Study of urinary urge correlations

As seen $n$ figure below (Figure 4), there is a close positive correlation between DB ranges and inflammation markers, respectively TNF-alpha $(r=0.9 ; p<0.0001)$ and CRP $(r=0.8$; $p<0.0001)$.

IBS is well known as intestinal disorder often associated to other functional pain conditions and various extraintestinal co-morbidities [14-16] Several pelvic floor conditions are reported by multiple studies as comorbidities associated to IBS: pelvic organ prolapse, urinary urgency, latency or incontinence

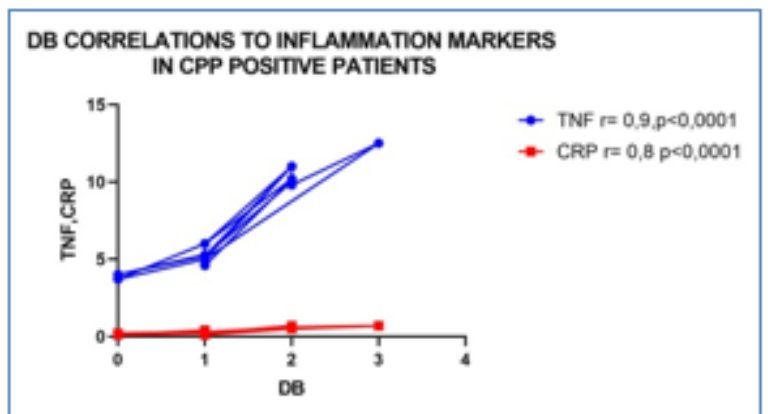

Fig. 4 Correlations between DB and inflammatory markers: CRP and TNF-alpha

$[17,18]$, as well as sexual dysfunctions[ 19,20$]$. Many researchers observed that there is a greater possibility of associated conditions like IBS, FM and. chronic fatigue syndrome in women with interstitial cystitis or painful bladder syndrome and also in those with higher levels of anxiety or depression [21]. The question whether these disorders have some common underlying pathways is still open to debates [22-24]. There is indeed a crosstalk between the bladder and bowel and a lot of overlap between CPP and overactive bladder symptoms $[25,26]$. There is also a complex physiology of urologic and gastrointestinal functions based on the convergence of dorsal root ganglia neurons, resulting in multiple pelvic organs sensors, located in diverse organs from pelvic area, such as: colon, bladder and gynecologic organs. Gut microbiota DB could alter this inter-organ dialogue by corruption of many pathways to the normal existing braingut connection. Moreover, it is by now demonstrated that gut DB could trigger inflammatory pathways that could manifest not only local, as an interstitial cystitis, in these cases, but also in different area, far from pelvic location $[27,28]$. In this view, our study observations showed positively good correlations between DB ranges and inflammatory markers, as well as between DB and all pelvic assessed symptoms, such as: urinary urge, dyspareunia and cystalgia. Based on these preliminary data, next question in line is: could probiotics represent a possible alternant tool to alleviate pelvic inflammation, triggered by gut DB? If these findings are validated by prospective studies, it could provide an interesting new perspective on the relationship IBS-CPP. 


\section{Conclusions}

Young female IBS patients with concurrent CPP symptoms often experienced other associated functional pain conditions like FM and migraine, along with anxiety, more severe abdominal complaints as well as higher gut DB and consecutively subclinical pro-inflammatory status. Strong positive correlations of gut DB to inflammatory markers as well as to CPP symptoms give the relationship IBS-CPP a new perspective.

\section{References}

1.DROSSMAN DA The functional gastrointestinal disorders and the Rome III process Gastroenterology, 130, 2006, p.1377

2.SPILLER R, CAMILLERI M, LONGSTRETH GF. Do the symptom-based, Rome criteria of irritable bowel syndrome lead to better diagnosis and treatment outcomes? Clin Gastroenterol Hepatol, 8,2010, p.125 3.CLOUSE RE, MAYER EA, AZIZ Q, et al. Functional Abdominal Pain Syndrome Gastroenterology,130(5),2006,p.1492

4.CRABTREE D, GANTY P, Common functional pain syndromes, BJA Education, 10( 16),2016, p.334

5.DUPONT HL Evidence for the role of gut microbiota in irritable bowel syndrome and its potential influence on therapeutic targets Aliment Pharmacol Ther, 39,2014,p.103

6.GUNTER J. Chronic pelvic pain: An integrated approach to diagnosis and treatment. Obstetrical and Gynecological Survey, 58(9),2003, p.615 7.ROME FOUNDATION. Guidelines-Rome III Diagnostic Criteria for Functional Gastrointestinal Disorders. J Gastrointestin Liver Dis., 15,2006,p.307

8.ENGELER D. BARANOWSKI A.P., BOROVICKA J . et al. Guidelines on chronic pelvic pain. European Association of Urology(EAU) uroweb.org/guideline/chronicpelvicpain, 2014

9.LIPTON RB, BIGAL ME, STEINER TJ et al. Classification of primary headaches Neurology. 63(3),2004,p.427

10.LIPTON RB, DOWSON AJ et al. Development and testing of the migraine disability assessment (MIDAS) questionnaire to assess headache related disability. Neurology, 56,2001,S20-S28

11.SPITZER RL, KROENKE K, WILLIAMSJBW, LOWE B. A brief measure for assessing generalized anxiety disorder. Arch Intern Med., $166,2006, p .1092$

12.DW ORKIN SF, LERESCHE L. Research diagnostic criteria for temporomandibular disorders: review, criteria, examinations and specifications, critique.J Craniomandib Disord., 6,1992,p.301

13.WOLFE F, CLAW DJ ., FITZCHARLES MA et al. The American College of Rheumatology Preliminary Diagnostic Criteria for Fibromyalgia and Measurement of Symptom Severity Arthritis Care \& Research, $69(5), 2010, p .600$
14. WHITEHEAD WE, PALSSON O, JONES KR. Systematic review of the comorbidity of irritable bowel syndrome with other disorders: what are the causes and implications? Gastroenterology, 122,2002, p.1140

15.GEORGESCU D, GEORGESCU LA. Chronic pelvic complaints in females with irritable bowel syndrome: another burden to deal with. ESI , 10(24), 2014, p.73

16.GEORGESCU D, REISZ D, GURBAN CV et al. Migraine in young females with irritable bowel syndrome: still a challenge Neuropsych Dis and Treat, 14,2018,p.21

17.MONGA AK, MARRERO J M, STANTON SL, LEMIEUX MC, MAXWELL JD. Is there an irritable bladder in the irritable bowel syndrome? $\mathrm{Br}$ J Obstet Gynaecol,104,1997, p.1409

18.J ELOVSEK JE, BARBER MD, PARAISO MF, WALTERS MD. Functional bowel and anorectal disorders in patients with pelvic organ prolapse and incontinence. Am J Obstet Gynecol, 193.2005,p.2105

19.GUTHRIE E, CREED FH, WHORWELL PJ. Severe sexual dysfunction in women with the irritable bowel syndrome: comparison with inflammatory bowel disease and duodenal ulceration. $\mathrm{Br}$ Med J (Clin Res Ed), 295,1987,p.577

20.FASS R, FULLERTON S, NALIBOFF B, HIRSH T, MAYER EA. Sexual dysfunction in patients with irritable bowel syndrome and non-ulcer dyspepsia. Digestion, 59,1998,p.79

21.WARREN JW, HOWARD FM, CROSS RK, et al. Antecedent nonbladder syndromes in case-control study of interstitial cystitis/ painful bladder syndrome. Urology, 73,2009, p. 52

22.RIEDL A, SCHMIDTMANN M, STENGEL A, et al. Somatic comorbidities of irritable bowel syndrome: a systematic analysis. J Psychosom Res, 64,2008,p.573

23.MIKOCKA-W ALUSA, TURNBULL D, Moulding N, Wilson I, Andrews JM, Holtmann G. Psychological comorbidity and complexity of gastrointestinal symptoms in clinically diagnosed irritable bowel syndrome patients. J Gastroenterol Hepatol, 23,2008,p.1137

24.WANG J, VARMA MG, CREASMAN J M et al. Pelvic Floor Disorders and Quality of Life in Women with Self-reported Irritable Bowel Syndrome. Alimentary Pharmacolog \&Therapeutics. ,31(3), 2010,p.424 25.KAPLAN SA, DMOCHOWSKI R, CASH BD, KOPP ZS, BERRIMAN SJ, KHULLAR V. Systematic Review of the Relationship Between Bladder and Bowel Function Implications for Patient Management.Int J Clin Pract., 67(3),2013,p.205

26.HEITKEMPER M., JARRETT M. Overlapping Conditions in Women With Irritable Bowel Syndrome .Urol Nurs.,25(1),2005,p.25

27.SHREINER AB, KAO JY, YOUNG VB. The gut microbiome in health and in disease. Curr Opin Gastroenterol ,31(1),2015,p. 69 28.YU LC, WANG JT, WEI SC, NI YH. Host-microbial interactions and regulation of intestinal epithelial barrier function: From physiology to pathology. World J Gastrointest Pathophysiol.,3(1),2012,p.27

Manuscript received: 21.08 .2019 